\title{
Single-shot two-dimensional multi-angle light scattering (2D-MALS) technique for nanoparticle aggregate sizing
}

\author{
Fabio J. W. A. Martins ${ }^{1}$ (1) $\cdot$ Andreas Kronenburg $^{2} \cdot$ Frank Beyrau $^{1}$
}

Received: 30 October 2020 / Accepted: 23 February 2021 / Published online: 17 March 2021

(C) The Author(s) 2021

\begin{abstract}
The two-dimensional multi-angle light scattering (2D-MALS) technique has been extended for single-shot size measurements of soot aggregates in flames. Six cameras are used for instantaneous acquisition of the elastic scattering from the aggregates at different directions between 10 to $90^{\circ}$ of a laser light sheet. Two diluted ethylene ( 50 and $60 \%$ by volume of $\mathrm{C}_{2} \mathrm{H}_{4}$ fuel diluted with inert $\mathrm{N}_{2}$ ) coflow laminar diffusion flames with little flickering are used as proof of concept. Results of instantaneous, average and fluctuating 2D fields of the effective radii of gyration, which are expected to characterize the size of the aggregates, compare well with the literature, demonstrating the applicability of the proposed sizing method to weakly unsteady combustion processes.
\end{abstract}

\section{Introduction}

In situ measurements of particle aggregate characteristics are crucial for a better understanding of physico-chemical mechanisms related to aggregate formation and evolution in combustion processes [3, 28, 41]. Although modelling and simulation of these complex formation mechanisms have matured significantly over the last years [3, 41], there are still considerable discrepancies between predicted and actual particle aggregate properties. The dissimilarities are in part a consequence of the lack of data and techniques capable of providing accurate measurements of the physical and chemical characteristics of particle aggregates during their development under a variety of combustion conditions. The existent ex situ tools are not suited for on-line monitoring and require extractive sampling, which perturbs the flow and chemistry under investigation $[14,35]$. In contrast, optical diagnostic techniques can assess relevant aggregate attributes in situ and non-invasively, such as particle sizes, morphology, composition and concentration [28]. These diagnostic techniques are suited for optimization and monitoring

Fabio J. W. A. Martins

fabio.martins@ovgu.de

1 Institute of Fluid Dynamics and Thermodynamics (ISUT), Otto von Guericke University Magdeburg, Magdeburg, Germany

2 Institute for Combustion Technology, University of Stuttgart, Stuttgart, Germany practical combustion systems. For example, many industrial combustors and engines frequently produce undesirable soot aggregates from incomplete combustion of hydrocarbon fuels and release them into the environment, causing detrimental effects on human health (e.g., lung, heart and vasculature) and on climate (e.g., global warming and melting of icebergs) $[4,16,25,48,50]$. The maximization of efficiency together with the reduction of soot emissions rely on the knowledge about the complex aggregate development, which can be gained from these type of measurements. This knowledge is paramount for the optimization and up-scaling of technical combustion systems for production of uncountable particle aggregate commodities with tailored properties [41, 49].

A variety of in situ optical techniques have been developed to characterize aggregate properties in the combustion [28]. Among them, line-of-sight attenuation (LOSA), sometimes termed light extinction, has been extensively used for point-wise and two-dimensional measurements of aggregate volume fraction [e.g., 7, 13, 11, 45]. This quantity can also be obtained through multi-wavelength information from aggregate radiative emission, referred to as spectral soot emission (SSE) techniques [e.g., 6, 26, 32]. Laserinduced incandescence (LII) has also been vastly employed to provide point measurements and planar fields of aggregate volume fraction as well as primary-particle size and, in combination with elastic light scattering (ELS), aggregate size [e.g., 37, 43, 42, 55, 29]. Elastic light scattering techniques based on few or multiple detectors (multi-angle 
light scattering, MALS) can estimate primary-particle size, aggregate size and morphology [e.g., 10, 38, 21, 36, 24]. For incipient aggregate particles (composed of few primary particles), small-angle X-ray scattering (SAXS) [e.g., 8, 15, 19] and small-angle neutron scattering (SANS) [e.g., 31, $53,58]$ can provide quantitative aggregate information. For more mature aggregate particles (tens to several hundreds of primary particles), wide-angle light scattering (WALS) [33, 34] or multi-angle light scattering [e.g., 10, 21, 24] are preferable to measure aggregate size and morphology in a pointwise manner. The latter ELS technique has been recently extended to two dimensions (2D-MALS) for measurements of the averaged aggregate characteristics [1, 20,27].

The elastic light scattering method has been already proved to be a powerful tool for aggregate particle sizing $[18,28,46]$. Until now, the approach has been mainly applied in two ways: (i) using a single one-dimensional detector [e.g., 10, 21, 24] or one camera sensor [e.g., 1, $20,27]$ mounted on a scanning goniometer, and (ii) using multiple one-dimensional fixed detectors [e.g., 36, 38] or a camera sensor imaging a wide-angle elliptical mirror [e.g., $33,34,17]$. The first methodology allows for averaged measurements of aggregate properties in one or two dimensions (according to the detector) in only stationary environments, due to the need of a time-consuming sequential signal acquisition for the various angular positions, whereas the second methodology allows for point-wise instantaneous measurements not only under steady but also unsteady environments, due to its simultaneous multi-angle signal registration.

In the present work, single-shot two-dimensional multiangle light scattering is introduced. The proposed approach overcomes limitations of previous ELS techniques as it combines the advantages of simultaneous signal acquisition with the capability of two-dimensional measurements. Therefore, instantaneous 2D fields of the scattered light signal ratio at various angles are less affected by changes in the flame conditions during the acquisition of the set and instantaneous quantities can be measured, allowing the evaluation of higher-order statistics within a plane as opposed to existing techniques that provide averaged quantities only. The present apparatus for synchronously signal recordings is composed by six camera sensors that are calibrated for their dissimilar optical setups based on the light scattering from pure gases. The performance of the proposed approach is demonstrated in $\mathrm{N}_{2}$-diluted 50 and $60 \% \mathrm{C}_{2} \mathrm{H}_{4}$ (with 50 and $40 \% \mathrm{~N}_{2}$ by volume, respectively) coflow laminar diffusion flames with small flickering. The measured averages and fluctuations of the effective radius of gyration fields of soot aggregates are compared and related to the literature.

\section{Theoretical background}

It is well-known that nearly spherical primary nanoparticles randomly aggregate and agglomerate forming fractal-like cluster structures in many combustion processes [3, 41, 49]. This section will describe the relevant concepts about elastic light scattering in the framework of fractals for the application of the present measurement technique. More details can be found in the reviews by Jones [18] and by Sorensen [46]. The fractal aggregate can be described as

$N_{p}=k_{f}\left(\frac{R_{\mathrm{g}}}{r_{p}}\right)^{D_{f}}$,

where $N_{p}$ is the amount of monodisperse primary particles forming the aggregate, $k_{f}$ is a proportionality factor generally in the range of $1.7-3.5[18,21,24], r_{p}$ is the radius of the primary particle, $D_{f}$ is the fractal dimension characterized by a non-integer number normally in the range of 1.4-2.2 $[18,21,46]$ and $R_{\mathrm{g}}$ is the radius of gyration, defined as

$R_{\mathrm{g}}=\sqrt{\frac{1}{N_{p}} \sum d_{p, i}^{2}}$,

where $d_{p, i}$ is the distance between the mass centre of the primary particle $i$ and the mass center of the aggregate. $R_{\mathrm{g}}$ is a representative characteristic of the aggregate size.

The relationship between scattered intensity and scattering angle is the most fundamental for size measurements of aggregates. The scattering wave vector, linked to the detection angle, is defined as the difference between the incident and scattered wave vector. Its magnitude $q$ is given by

$q(\lambda, \theta)=\frac{4 \pi}{\lambda} \sin \left(\frac{\theta}{2}\right)$,

where $\lambda$ is the light wavelength used in the measurements and $\theta$ is the angle between the incident and scattered wave vector.

The light scattered by fractal aggregates can be interpreted by the Rayleigh-Debye-Gans theory (RDG) [18, 46], within $10 \%$ of accuracy for $D_{f} \simeq 1.8$ [46]. The scattered intensity $I_{\text {sca }}$ collected by a sensor (assuming monodisperse distribution from both aggregates and primary particles, and independent scattering signals from primary particles) can be described as

$I_{\text {sca }}(q)=\alpha I_{i n c} n_{a} N_{p}^{2} V \frac{d \sigma^{p}}{d \Omega} S\left(q R_{\mathrm{g}}\right)$,

where $\alpha$ is a proportionality factor related to the collection efficiency of the detection system, $I_{\text {inc }}$ is the incident light irradiance, $n_{a}$ is the aggregate number density, $V$ is the probe volume, $d \sigma^{p} / d \Omega$ is the differential scattering cross-section of the primary particle and $S\left(q R_{\mathrm{g}}\right)$ is the optical structure factor of the aggregate. 
The behaviour of the optical structure factor with respect to $q R_{\mathrm{g}}$ can be approximated in three different regimes as

$S\left(q R_{\mathrm{g}}\right) \quad=1 \quad q R_{\mathrm{g}} \ll 1 \quad$ (Rayleigh regime)

$S\left(q R_{\mathrm{g}}\right) \cong 1-\frac{1}{3} q^{2} R_{\mathrm{g}}^{2} \quad q R_{\mathrm{g}} \leq 1 \quad$ (Guinier regime)

$S\left(q R_{\mathrm{g}}\right)=\beta\left(q R_{\mathrm{g}}\right)^{-D_{f}} \quad q R_{\mathrm{g}}>1 \quad$ (Power law regime)

where $\beta$ is an empirical proportionality factor around unity [46].

In the Guinier regime, an effective radius of gyration can be estimated from the angular coefficient of the linear curve fitting from the scattered light intensity ratio as a function of the square of the scattering wave vector. To this end, the ratios of scattered light intensities are obtained from Eq. (6) by the aid of a Taylor expansion:

$\frac{I_{\text {sca }}(\lambda, 0)}{I_{\text {sca }}(\lambda, \theta)}=\frac{S(0)}{S\left(q R_{\mathrm{g}, \text { eff }}\right)} \approx 1+\frac{1}{3} R_{\mathrm{g}, \mathrm{eff}}^{2}(q(\lambda, \theta))^{2} \quad q R_{\mathrm{g}, \mathrm{eff}} \leq 1$

where $R_{\mathrm{g}, \mathrm{eff}}$ is the effective radius of gyration.

An actual aggregate population is generally composed of polydisperse distributions of both primary particles and aggregates. Therefore, the measured radius of gyration (output of the present technique) is an effective value representing the ensemble of the polydisperse aggregate [27, 33, 46]. The value is a weighted average associated with the distributions of the number of primary particles in the aggregates, aggregate number density and radius of gyration of aggregates. The effective radius of gyration can be mathematically defined as [27]

$R_{\mathrm{g}, \mathrm{eff}}=\sqrt{\frac{\int N_{p}^{2} R_{\mathrm{g}}^{2}\left(N_{p}\right) n_{a}\left(N_{p}\right) d N_{p}}{\int N_{p}^{2} n_{a}\left(N_{p}\right) d N_{p}}}$.

It is possible to observe from this equation that the $R_{\mathrm{g}, \text { eff }}$ value is larger than the arithmetic mean value, because the scattered light signal is dominated by larger aggregates [20, $27,33]$. Therefore, this fact must be taken into account for a meaningful comparison of statistics between measured effective radii of gyration by MALS techniques and radii of gyration from sampled aggregates or from computational simulations, for example.

\section{Experimental setup}

Two diluted- $\mathrm{C}_{2} \mathrm{H}_{4}$ non-sooting coflow laminar diffusion flames are investigated in the present work. Here the term 'non-sooting' refers to the absence of soot aggregates downstream of the flame closed tip due to soot-oxidation process, despite the presence of soot at upstream locations [22]. The flames are produced in a burner with a 4-mminner 6-mm outer-diameter vertical tube centralized in a $44 \times 70-\mathrm{mm}^{2}$ rectangular duct. The diluted fuel is a mixture of 50 and $60 \% \mathrm{C}_{2} \mathrm{H}_{4}$ (ethylene) by volume with 50 and $40 \% \mathrm{~N}_{2}$, respectively. A mixture of $21 \% \mathrm{O}_{2}$ and $79 \% \mathrm{~N}_{2}$ by volume is used to emulate air in the coflowing stream through the rectangular duct, because compressed air was not available. The coflow oxidizer stream supports the flame stabilization, attenuating environmental fluctuations. The measurements are taken after about $5 \mathrm{~min}$ when the burner is thermalized and the flow conditions are statistically stationary. The gas flow rates, fixed in both flames, are controlled by Bronkhorst mass flow meters within uncertainties below $3 \%$. The flow rates of fuel and oxidizer are $0.18 \mathrm{~L} / \mathrm{min}$ and $63.88 \mathrm{~L} / \mathrm{min}$ under $1 \mathrm{~atm}$ and $301 \pm 2 \mathrm{~K}$, respectively. The fuel velocity at the pipe exit displays a parabolic profile with a bulk velocity of $0.24 \mathrm{~m} / \mathrm{s}$, while the coflowing gas has an approximately constant velocity of $0.35 \mathrm{~m} / \mathrm{s}$, obtained using a packed bed of spheres and a honeycomb. For a better optical access, no housing is used around the burner. The burner exhaustion is collected by a chimney hood located about $1.5 \mathrm{~m}$ above the measurement region. The burner is fixed upon a motorized table, which can be used to explore different regions of interest (ROI).

The experimental setup is presented in Fig. 1. A singlepulse frequency-doubled Nd:YAG laser (Quantel YG980, maximum energy $820 \mathrm{~mJ} @ 10 \mathrm{~Hz}$ ) operating at 532-nm wavelength is employed. The laser power is controlled by a combination of half-wave plate and a polarizing beamsplitter cube (Thorlabs PBS25-532-HP), while the laser output energy is kept constant at $150 \pm 4 \mathrm{~mJ}$. This system allows for energy adjustment with minor variations in the beam profile and ensures vertical polarization in the measurement region (polarization ratio better than 3000:1). A power meter (Coherent LabMax-Top), located at the end of the optical path, monitors the laser light power. The light sheet, passing through the burner diametral plane, is formed by a $-50-\mathrm{mm}$ cylindrical lens followed by a 500-mm plano-convex lens. A minimum smearing effect at oblique detection angles is observed in the present measurements. The smearing can appear as a consequence of imaging onto different pixels the scattering signal from aggregates at the same radial location in the flame but at a different position along the light sheet thickness [27]. A circular aperture diaphragm is used between the two lenses to crop the upper and lower edges of the light sheet, avoiding spurious reflections at the latter lens rim and removing regions with lower laser fluence. The formed laser sheet has an approximate constant height of $36 \mathrm{~mm}$. The scattered light is imaged by six CCD cameras (LaVision Imager proX, $1600 \times 1200$ pixel sensor, $7.4 \mu \mathrm{m}$ pixel pitch, 14 bit) positioned in a circular arrangement 

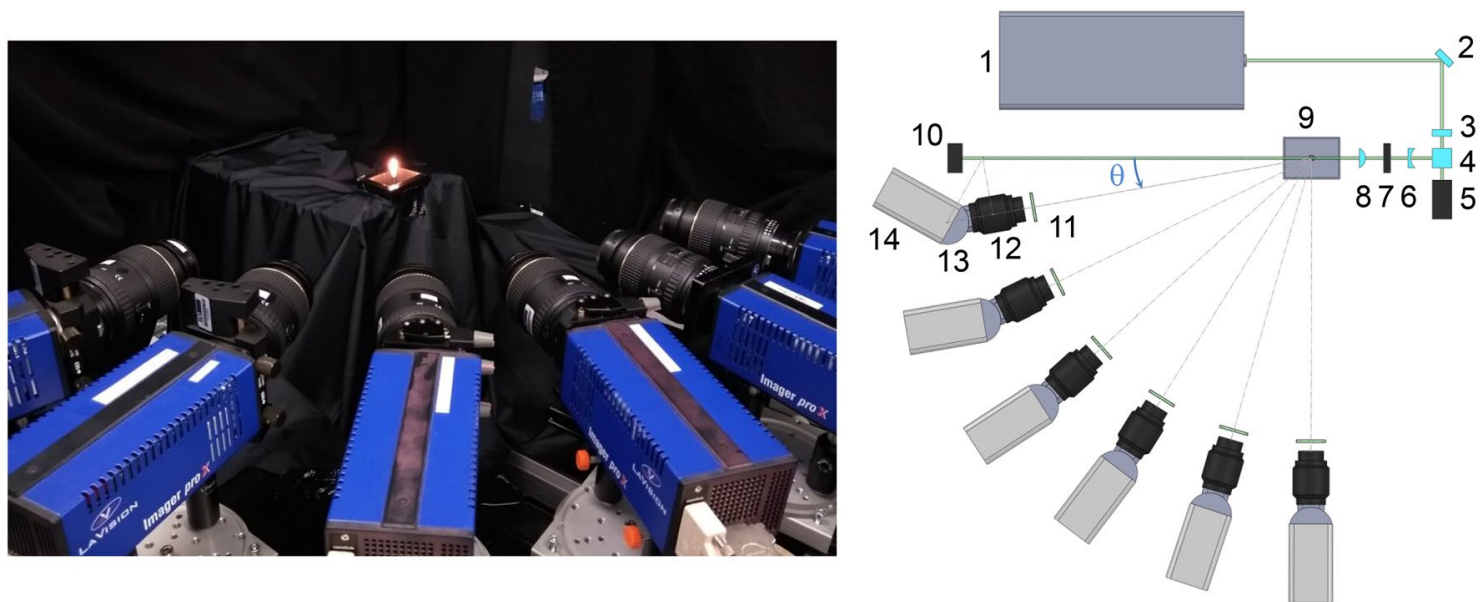

Fig. 1 Single-shot 2D-MALS arrangement using six cameras for in situ planar-measurements of the instantaneous effective radii of gyration. Legend: (1) Nd:YAG laser, (2) mirror, (3) half-wave plate, (4) polarizing beamsplitter cube, (5) beam dump, (6) cylindri-

around the burner from $\theta=10^{\circ}$ to $90^{\circ}$ (angles measured with respect to the forward propagation direction of the incident light) with $16^{\circ}$ angular space between adjacent cameras. The selected camera positions are a compromise between the sensitivity for small aggregates (necessity of large $q$-values) and the applicability limits of the RDG approximation $\left(q R_{\mathrm{g}} \leq 1\right.$ and $\left.I_{\mathrm{sca}}(0) / I_{\mathrm{sca}}(\theta) \leq 2\right)$. The cameras are equipped with 100-mm objective lenses (Tokina ATX Pro D Macro) and positioned at about $500 \mathrm{~mm}$ from the burner centre, leading to a vertical magnification close to 0.2 . The magnification adopted allows for the registration of the entire flame soot region in a single-shot picture. Scheimpflug adapters are used to obtain sharp images in the entire ROI. Flame luminescence is strongly suppressed by a 532-nm bandpass filter with 10-nm bandwidth mounted in front of the objective lens in combination with a short sensor exposure time. The system is triggered by a programmable time unit controlled by Davis 8.4 software (LaVision). The images are acquired at $10 \mathrm{~Hz}$ with the laser light pulse (about $12 \mathrm{~ns}$ ) centred at the $0.5-\mu$ s sensor exposure time. The scattering signal from aggregates are acquired with a low laser fluence below $0.2 \mathrm{~J} / \mathrm{cm}^{2}$ to prevent alteration in the soot morphology [22, $51,57]$ or influence by possible LII signal [29]. Blackout materials are used in the surroundings to minimize stray light.

The objective apertures and sensor binning are adjusted taking into account the collected scattered signal and the spatial resolution, both related to the camera viewing direction (detection angle). The signal magnitude is proportional to the probe volume, which at a detection angle $\theta$ is a factor of $1 / \sin (\theta)$ greater than that at $90^{\circ}$ [27]. The objective apertures are adjusted to $f / 5.6$ for cameras at $10^{\circ}, 26^{\circ}$ and $42^{\circ}$, and they are adjusted to $f / 4$ for the others. The sensor binning is cal lens, (7) aperture, (8) plano-convex lens, (9) burner, (10) power meter, (11) bandpass filter, (12) lens, (13) Scheimpflug adapter and (14) CCD camera

set to $1 \times 2$ (horizontal $\mathrm{x}$ vertical) for all cameras, leading to a minimum spatial resolution of 6 pixel $/ \mathrm{mm}$ at $10^{\circ}$ sensor. Due to the perspective, the images become narrower along the horizontal direction for smaller detection angles by a factor of $\sin (\theta)$. The acquired scattered signals are always between $15 \%$ and $90 \%$ of the full sensor range, to obtain a good signal-to-noise ratio and to operate the sensor in its linear region. The highest signals are recorded at the flame wings for the $60 \% \mathrm{C}_{2} \mathrm{H}_{4}$ diffusion flames (Fig. 3c).

\section{Signal processing}

The image processing and evaluation procedure, very important to extract meaningful sizing measurements from the 2D-MALS signal, will now be described. The signal processing is performed using Matlab R2019b software. The procedure steps (flowchart illustrated in Fig. 2) can be summarized as: (1) noise removal, (2) background correction, (3) optical setup correction, (4) spatial correction, and (5) radius of gyration evaluation. Figure 3 presents these steps applied on a typical light scattering image from soot aggregates.

First, salt-and-pepper noise, sometimes present in the raw scattering images $I_{\text {raw }}$ (Fig. 3c), is removed by a $3 \times 3$ median filter as follows

$I_{\text {raw }}^{\text {filt }}(u, v, \theta, t)=\operatorname{median}\left\{I_{\text {raw }}(u+i, v+j, \theta, t)\right\}$,

where $u$ and $v$ are the camera coordinates, $\theta$ is the detection angle in the image stack, $t$ is the time instant and $i$ and $j$ denote integer indexes varying from -1 to 1 . 


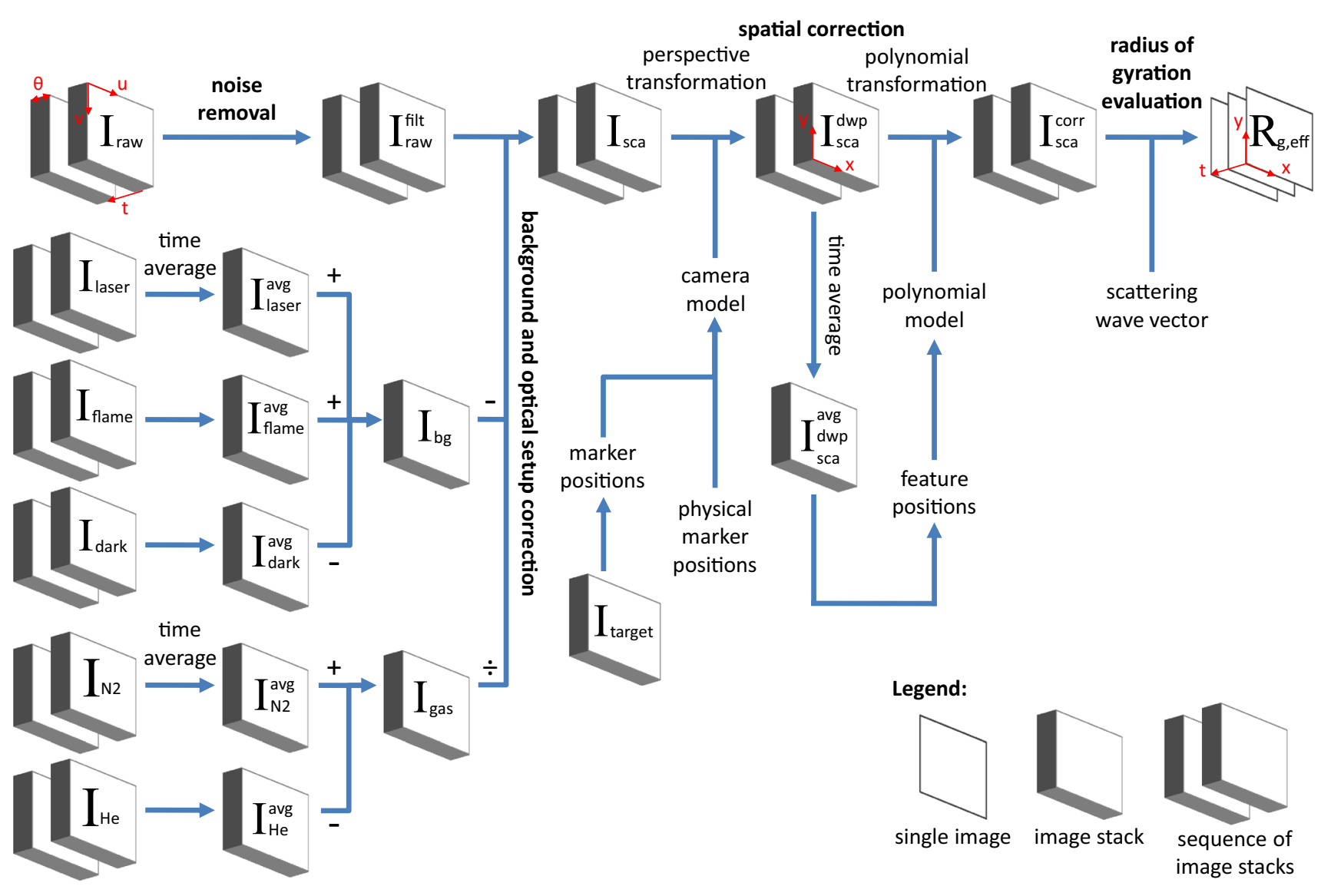

Fig. 2 Flowchart of the image processing and evaluation procedure steps for instantaneous size measurements using single-shot 2D-MALS

Second, a background image (Fig. 3a) is subtracted from the instantaneous scattering images to correct for stray laser light (from scattering by optical components along the laser beam path and reflections on the surfaces), ambient light, flame luminescence and camera noise (from thermal noise, readout noise and differences in A/D converters). Following Altenhoff et al. [1], the background image $I_{\mathrm{bg}}$ is generated for each camera viewing direction based on averaged images of the dark background $I_{\text {dark }}^{\text {avg }}$ (without flame and laser), the laser background $I_{\text {laser }}^{\text {avg }}$ (without flame, but with laser on) and the flame background $I_{\text {flame }}^{\text {avg }}$ (with flame, but without laser) according to

$I_{\mathrm{bg}}(u, v, \theta)=I_{\text {laser }}^{\mathrm{avg}}(u, v, \theta)+I_{\text {flame }}^{\mathrm{avg}}(u, v, \theta)-I_{\text {dark }}^{\mathrm{avg}}(u, v, \theta)$.

Each averaged image is calculated from 100 images. It is important to mention that the flame luminescence, which is low in the present measurements (see Fig. 3a) due to the use of bandpass filter and small exposure time, can only be properly corrected in instantaneous light scattering measurements when the flame is steady.

Third, the optical setup must be calibrated since the actual characteristics of each detector can differ from those stated by the manufacturer. The optical setup calibration accounts for differences in the imaging system (such as differences in the sensor quantum efficiencies, light collection efficiencies, lenses, bandpass filters, apertures, Scheimpflug tilt angles, hardware-binned pixel sizes, magnifications, AD conversion factors), as well as the angledependent probe volume, aberrations from the optical components and inhomogeneities in the laser light sheet (spatial variation in the laser fluence). To this end, a calibration image is computed based on the light scattering from gases [1, 21, 33, 36]. The scattered light from gas molecules irradiated by vertically polarised light, which is in the Rayleigh regime, must be isotropic (Eq. 5). As a consequence, the non-uniformities in the recorded calibration image are linked to the optical setup. An averaged image $I_{N_{2}}^{\text {avg }}$ of 150 light scattering images from pure nitrogen, flowing through the entire burner, is used to calibrate the optical setup. To increase the recorded signal in each image, a long frame exposure is used to integrate over the light scattering from 80 laser pulses. For the time-average computation, fluctuating intensity values above 3 standard deviations within the pixel history are filtered out to 

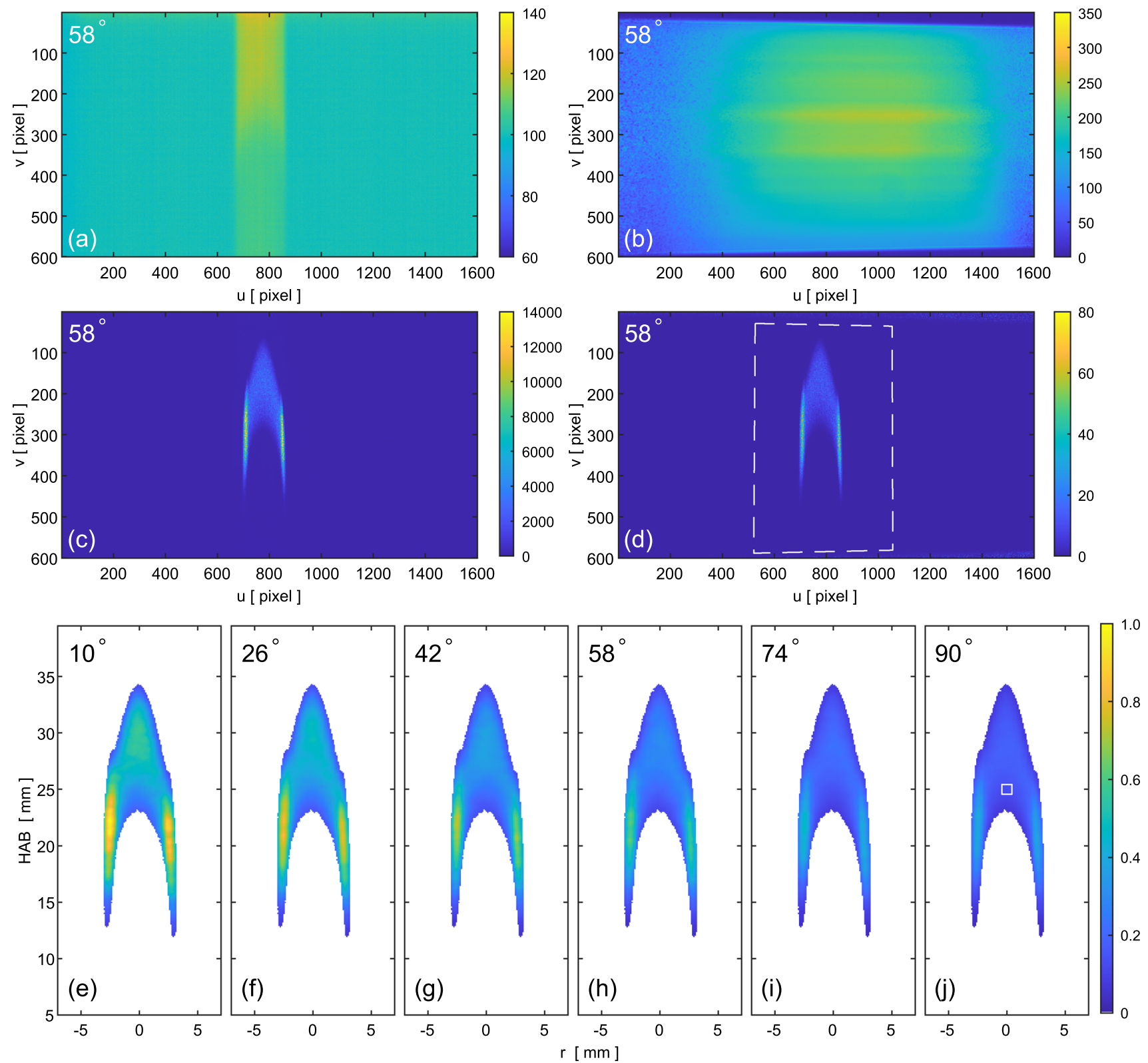

Fig. 3 Processing steps on a typical instantaneous image of the light scattering from soot aggregates in the diluted $60 \% \mathrm{C}_{2} \mathrm{H}_{4}$ diffusion flame for the $58^{\circ}$ direction: a background image, b optical-setup image, $\mathbf{c}$ single-shot raw image and $\mathbf{d}$ background-corrected image

divided by optical-setup image with undistorted region of interest (dashed white polygon). $\mathbf{e}-\mathbf{j}$ Dewarped signal images normalized by the maximum of the set and masked by the flame shape for different viewing directions. In all images the laser irradiates from right to left

remove possible scattering from isolated dust particles that inevitably entrain in the outer region of the flow. Additionally, the calibration offset (from stray laser light, ambient light and camera noise, for example) is considered based on the light scattering from pure helium, which has a negligible molecule cross-section compared to that of nitrogen $[2,33]$. An averaged image $I_{H e}^{\text {avg }}$ is calculated from 150 helium scattering images, also using 80 laser pulses within

a long frame exposure. Therefore, the calibration image $I_{\text {cal }}$ (Fig. 3b) is computed subtracting the averaged images of Rayleigh scattering from gases:

$I_{\text {cal }}(u, v, \theta)=I_{N_{2}}^{\mathrm{avg}}(u, v, \theta)-I_{H e}^{\mathrm{avg}}(u, v, \theta)$.

The corrected instantaneous image of scattering signal $I_{\text {sca }}$ (Fig. 3d) is obtained subtracting the median-filtered raw 
image $I_{\text {raw }}^{\text {filt }}$ by the background image and then dividing by the optical-setup calibration image, that is

$I_{\mathrm{sca}}(u, v, \theta, t)=\frac{I_{\mathrm{raw}}^{\mathrm{filt}}(u, v, \theta, t)-I_{\mathrm{bg}}(u, v, \theta)}{I_{\mathrm{cal}}(u, v, \theta)}$.

Forth, to evaluate the radius of gyration in two dimensions, the scattering images must be spatially coincident, so the intensity ratio can be computed on a pixel basis. In the present work, the scattering images are dewarped by Homography transformations (camera pinhole model) [12], correcting for the perspective of the measured plane due to the tilted camera viewing directions (cf. Figs. $3 \mathrm{~d}$ and $3 \mathrm{e}-\mathrm{j}$ ). To this end, a calibration plate composed by dot markers in a $5 \times 5 \mathrm{~mm}$ Cartesian grid is employed as a target (Fig. 4). It is placed at the ROI, aligned with the laser light sheet. The dot centres from the calibration images $I_{\text {target }}$ are automatically detected based on the centroid. A homography matrix $\mathbf{M}_{\theta}$ for each camera is then obtained from the correspondence between the dot markers in the image and those in the physical space $(x, y)$ applying the Direct Linear Transformation (DLT) [12]. The planar homography transformation, using homogeneous coordinates, is defined as

$\left[\begin{array}{c}x_{i} \\ y_{i} \\ 1\end{array}\right]=\frac{1}{k_{i, \theta}} \boldsymbol{M}_{\theta}\left[\begin{array}{c}u_{i, \theta} \\ v_{i, \theta} \\ 1\end{array}\right]=\frac{1}{k_{i, \theta}}\left[\begin{array}{ccc}1 / s & 0 & 0 \\ 0 & 1 / s & 0 \\ 0 & 0 & 1\end{array}\right]\left[\begin{array}{lll}m_{1, \theta} & m_{2, \theta} & m_{3, \theta} \\ m_{4, \theta} & m_{5, \theta} & m_{6, \theta} \\ m_{7, \theta} & m_{8, \theta} & 1\end{array}\right]\left[\begin{array}{c}u_{i, \theta} \\ v_{i, \theta} \\ 1\end{array}\right]$,

where $k$ is a normalization factor for the homogeneous coordinate that depends on the marker $i$ at the camera position $\theta$, $s$ is a scaling factor to convert pixel coordinates into physical coordinates and $m$ are entries of the homography matrix related to the camera parameters. The scaling factor dictates the final spatial resolution of the dewarped images. In the present work, $s=9.3 \mathrm{pixel} / \mathrm{mm}$ is adopted based on the calibration markers in the images and in the physical space. The dewarped instantaneous scattered image $I_{\text {sca }}^{\text {dwp }}$ results from the perspective transformation using the calculated homography matrix entries according to
$I_{\mathrm{sca}}^{\mathrm{dwp}}(x, y, \theta, t)=\operatorname{transform}\left\{I_{\mathrm{sca}}(u, v, \theta, t)\right.$, perspective $\}$.

The transformation function is readily available in the Matlab Image Processing toolbox for a given correspondence of points. The physical coordinate $(x, y)$ is sometimes termed in the plots as $(r, \mathrm{HAB})$ in reference to the radial direction and height above the burner, respectively.

Although this simple camera model does not consider lens aberrations or strong image distortions (e.g. barrel and cushion types), both are negligible in the present measurements as observed in Fig. 4. The present calibration images show dot markers with expected elliptical shape due to the perspective projection of the round dots and excellent linear alignments among markers at the same abscissa and those at the same ordinate (keeping in mind that angles between lines are not preserved in a perspective view) [12]. Excellent spatial coincidence is obtained among the corresponding points using the employed camera model, quantified by a root mean square error of 0.11 pixel and a maximum projection error of 0.5 pixel (Fig. 5). The maximum projection error is observed along the horizontal direction for the $10^{\circ}$ view and was anticipated due to the shrinkage of the calibration plate in this camera image (Fig. 4). Projection errors below half a pixel are desirable to minimize possible spatial mismatch among dewarped images that could bias the scattering signal ratios and consequently the effective radius of gyration values.

In many cases, the initial image transformation needs to be further corrected for (i) misalignment between the calibration plate and the light sheet and for (ii) refractive index fluctuations. Frequently, the calibration plate is not perfectly aligned or it is slightly tilted relative to the light sheet (measurement plane), therefore the dewarped scattering images end up not being spatially coincident $[39,54,56]$. Additionally, the refractive index can fluctuate dynamically along the scattering and illumination paths under combustion processes [40, 47, 52]. Chemical reactions during the combustion locally change the gaseous mixture composition, temperature and density, directly influencing in the refractive index. Therefore, patterns in the scattering images can be
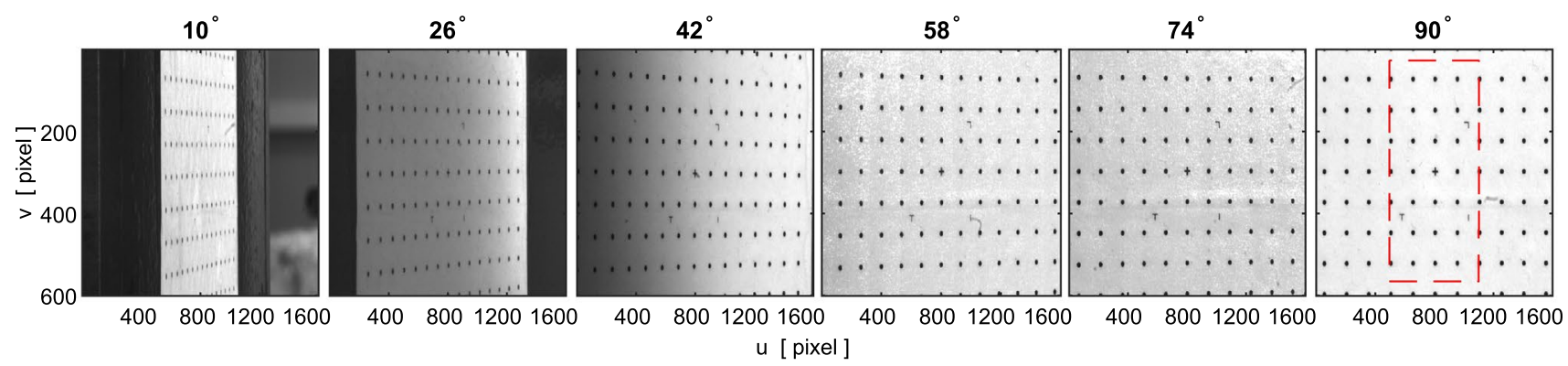

Fig. 4 Acquired images of a calibration plate for the six viewing directions. Region of interest is overlaid as dashed red polygon in the $90^{\circ}$ direction 


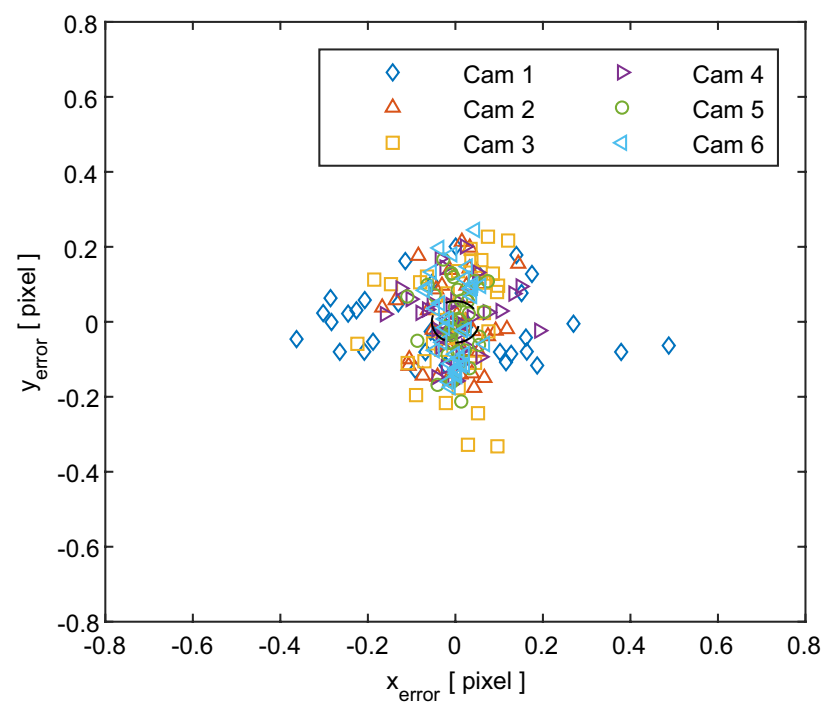

Fig. 5 Projection errors along $x$ and $y$ directions between corresponding points used for calibration of the six cameras. The root mean square error is marked with a dashed line

subjected to deformation and blur, due to light deflection by the inhomogeneous refractive index, which can not be sufficiently corrected by the initial geometric mapping based on a calibration plate surrounded by air at ambient temperature. The problem is worst for cameras at low detection angles and for measurements under high-temperature flames. In the present measurements, the flames are statistically stable with very little flickering. Therefore, a minor additional spatial correction based on matching the flame morphology of dewarped scattering images is enough to remedy these problems. The additional corrections also fix common horizontal offsets between the scattering images (below 15 pixels in the present work), which hinder a pixel-wise measurement. Corresponding points (features) are extracted at specific positions along the enlarged flame boundary and the skeleton of the time-averaged dewarped scattering signal image for each camera direction (Fig. 6), because of their similar relative spatial variation of the scattering dewarped signal intensity (Eq. 4). The flame boundary is obtained thresholding the scattered signal image, while the skeleton is computed from the intensity gradient. Corresponding point locations are derived based on the extreme positions of skeleton lines (dashed curves in Fig. 6) and their interception position on the flame centreline. The additional correction is then obtained by a 2 nd-order polynomial transformation for each camera as

$x_{i}^{\prime}=a_{1, \theta}+a_{2, \theta} x_{i, \theta}+a_{3, \theta} y_{i, \theta}+a_{4, \theta} x_{i, \theta} y_{i, \theta}+a_{5, \theta} x_{i, \theta}^{2}+a_{6, \theta} y_{i, \theta}^{2}$

$y_{i}^{\prime}=b_{1, \theta}+b_{2, \theta} x_{i, \theta}+b_{3, \theta} y_{i, \theta}+b_{4, \theta} x_{i, \theta} y_{i, \theta}+b_{5, \theta} x_{i, \theta}^{2}+b_{6, \theta} y_{i, \theta}^{2}$
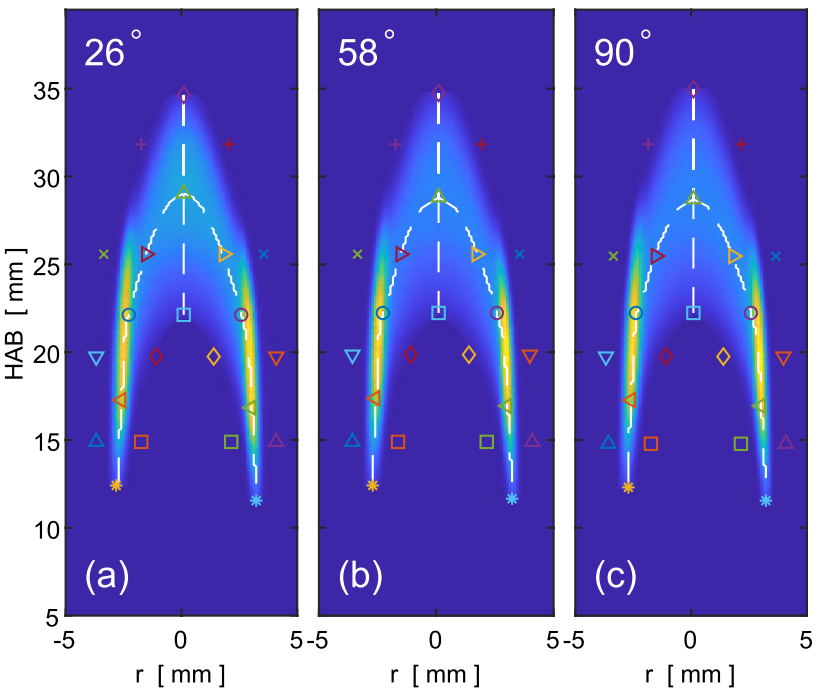

Fig. 6 Corresponding control points (symbols) for extra spatial correction extracted from time-averaged dewarped images at a $26^{\circ}, \mathbf{b} 58^{\circ}$ and $\mathbf{c} 90^{\circ}$ directions. The intensity is normalized in each image to enhance visualization. Computed flame skeleton based on the signal intensity is displayed as dashed white curves

where the quotation mark represents the transformed coordinate position and $a$ and $b$ are coefficients computed assuming the points at $90^{\circ}$ direction as target positions, i.e. $\left(x_{i}^{\prime}, y_{i}^{\prime}\right)=\left(x_{i, 90^{\circ}}, y_{i, 90^{\circ}}\right)$.

In the present work, the additional correction employed is the same for all instantaneous flames with the same dilution and it can be expressed as

$I_{\text {sca }}^{\text {corr }}(x, y, \theta, t)=\operatorname{transform}\left\{I_{\text {sca }}^{\mathrm{dwp}}(x, y, \theta, t)\right.$, polynomial $\}$,

where the polynomial transformation employs the computed coefficients $a$ and $b$ for each viewing direction. The root mean square errors for these transformations are below 0.7 pixel (i.e. $0.08 \mathrm{~mm}$ ). Contrarily, for other measurements under high temporal variations of refractive index along the viewing directions (e.g., high-temperature turbulent flames), instantaneous spatial corrections are necessary for singleshot measurements.

Finally, the evaluation of an instantaneous two-dimensional field of the radius of gyration is based on ratios of the dewarped scattering fields according to Eq. 8, after the image processing steps aforementioned. The use of instantaneous signal ratios instead of absolute quantities makes the approach robust against temporal changes in the flame conditions and inevitable pulse-to-pulse fluctuations in the laser energy. Since two-dimensional scattered signal at rigorously $0^{\circ}$ detection angle is not feasible as the projected area equals zero, the value is usually generated from the extrapolation of scattering fields of smallest angles [1, 21, 24, 34] or from using the scattering field at the lowest detection angle of $10^{\circ}$ 
instead $[20,27,33]$. The latter approach tends to bias the estimated values towards smaller radii of gyration, increasing the error [27, 33]. In the present analysis, the scattered signal at $0^{\circ}$ is computed from a 2 nd-order extrapolation using the three smallest-angle scattering fields. The scattering wave vector is assumed constant for the entire camera sensor at each detection angle (i.e. parallel light rays), due to the much larger camera distance compared to the size of the ROI. The additional relative error in the angle, and consequently in the scattering wave vector magnitude, is less than $2 \%$ using this assumption. Local averaged values within a $7 \times 7$ kernel (white square in Fig. 3j) are employed in each scattering image for the calculation of the scattering ratio at each point. The dynamic spatial range (DSR), ratio between ROI and spatial resolution, is above 100 for the present measurements. Figure 7 presents examples for the evaluation of the effective radius of gyration in three points in the measurement field. Following Eq. 8, the instantaneous 2D field of effective radius of gyration is extracted from the slope of a least-squares line fitting using those scattering ratios versus the corresponding square of the scattering wave vector magnitudes as

$\frac{I_{\text {sca }}\left(x, y, 0^{\circ}, t\right)}{I_{\text {sca }}(x, y, \theta, t)} \approx p_{0}(x, y, t)+p_{1}(x, y, t)(q(\theta))^{2}$,

where $p_{0}$ and $p_{1}$ are fitting coefficients, and the effective radius of gyration is

$R_{\mathrm{g}, \mathrm{eff}}(x, y, t)=\sqrt{3 p_{1}(x, y, t)}$.

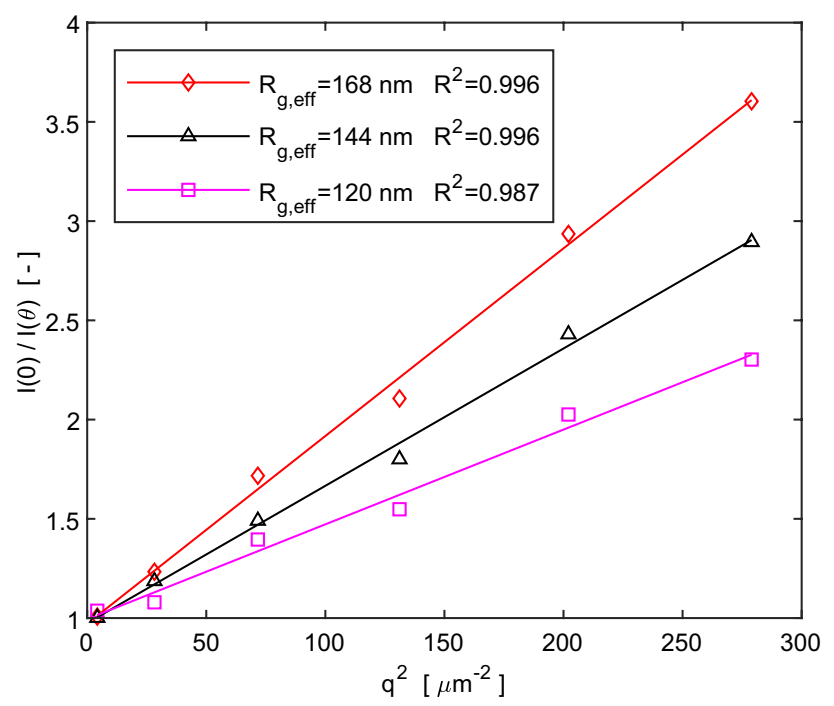

Fig. 7 Examples of line fittings for effective radius of gyration computation using single-shot 2D-MALS
Following Sorensen [46], the Guinier linear fit is only recommended for values of $I(0) / I(\theta) \leq 2$ (which is slightly above $\left.q R_{\mathrm{g}} \leq 1\right)$. Nevertheless, it is acceptable to stretch this limit for Guinier analysis of fractal aggregates with polydispersity driven by the aggregation kinetics, according to the same author. In these polydisperse systems, linear dependency can extend far beyond this limit, such as in the case of Titania aerosol [46] and soot aggregates from ethylene laminar diffusion flames [27]. For the present demonstration, we evaluate the effective radius of gyration using $I(0) / I(\theta)<4$ since good linearity is observed (Fig. 7). This minimizes discontinuities in the radius of gyration fields and allows for a more robust evaluation with more viewing directions included. The differences between the present evaluation with that using a more conservative limit are estimated to be below $5 \%$ for the investigated flames of the current work. For future measurements, the camera positions can be optimised according to the maximum expected effective radius of gyration of the system.

\section{Results and discussion}

The proof of concept of the present single-shoot 2D-MALS approach will now be demonstrated in 50 and $60 \% \mathrm{C}_{2} \mathrm{H}_{4}$ diluted coflow laminar diffusion flames with small flickering, where fluctuations of the aggregate morphology is expected in space and time. The flickering can be roughly estimated from the fluctuation of the flame shape height, obtained from the scattered signal (Fig. 6). The standard deviation of the height from the present 50 and $60 \% \mathrm{C}_{2} \mathrm{H}_{4}$ diffusion flames are 4.8 pixels $(0.51 \mathrm{~mm})$ and 1.5 pixels $(0.16 \mathrm{~mm})$, respectively. The presence of flame flickering is a result of buoyancy-induced instabilities, due to combustion, combined with oscillations at the surroundings, because the present coflowing stream is insufficient to fully shield the inner flow. Both flames display a cone-like shape, which is entirely registered in single-shot pictures.

Figure 8 presents instantaneous effective radius of gyration fields and $R^{2}$ fields of the least-squares fitting from the $60 \% \mathrm{C}_{2} \mathrm{H}_{4}$ diffusion flame at three random times (named as $t 1, t 2$ and $t 3$ ). The measurement region is restricted to locations with sufficient signal intensity. Instantaneous flame masks, based on overlaid transformed scattering images, are used to filter out measurements. Typical range and spatial distribution of soot aggregate sizes can be observed in the two-dimensional measured fields (Fig. 8a-c). The present single-shot 2D-MALS provides measurements of effective radius of gyration between 80 and 180 . Regions of radius of gyration below 80 are not detected due to the lower scattering signal (revisit Fig. 3c). This is a result of the small aggregate sizes combined with a low amount of these aggregates (Eq. 4). Interestingly, the highest scattering signals at 

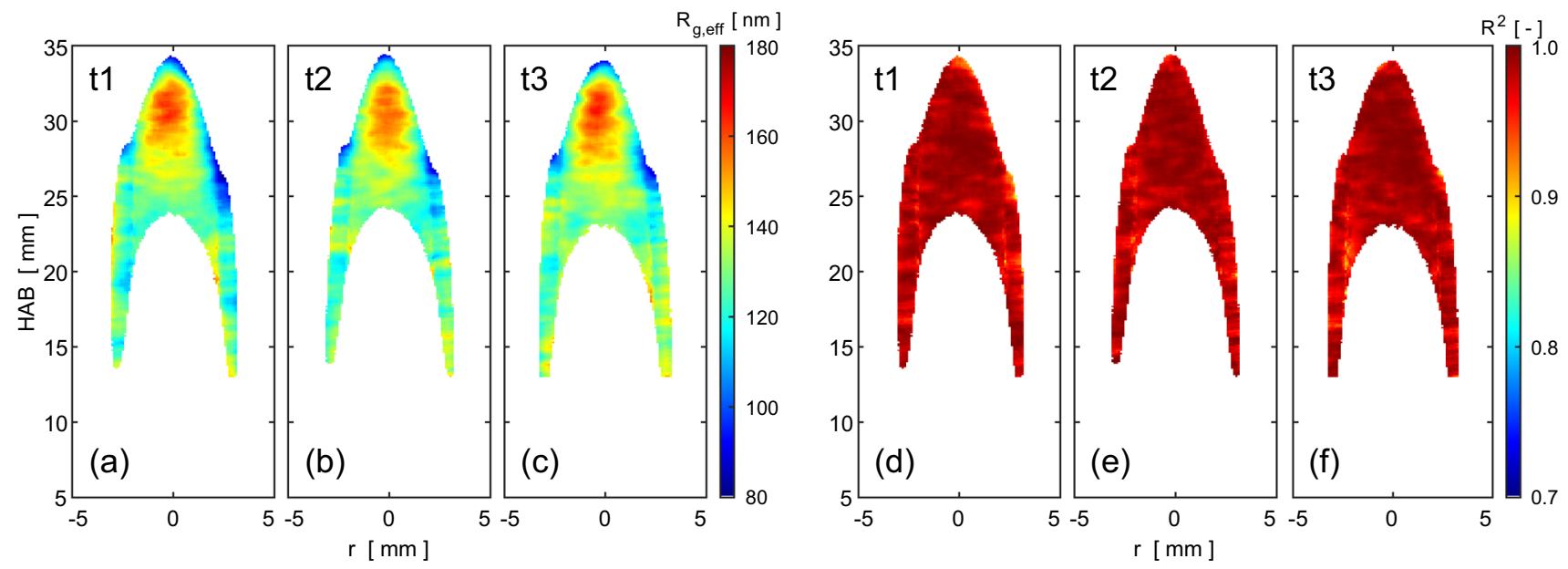

Fig. 8 Examples of single-shot two-dimensional instantaneous fields of $(\mathbf{a}-\mathbf{c})$ effective radius of gyration and $(\mathbf{d}-\mathbf{f})$ corresponding $R^{2}$ of the line regressions for the diluted $60 \% \mathrm{C}_{2} \mathrm{H}_{4}$ diffusion flame

the upper region of the flame wings do not correspond to the highest effective radii of gyration. This is expected due to the high soot concentration in these regions. The soot inception occurs at the fuel-rich areas at the centreline region and wings of the diffusion flame, and subsequently surface growth and aggregation happen [44]. Since the inception process at the wings occurs earlier upstream compared to that at the centreline region, more aggregates are formed at the former region and then advected towards the flame centreline, increasing the concentration at the upper wing region.

The uncertainty of effective radius of gyration by singleshot 2D-MALS is expected to be about $10 \%$ around the flame centreline, similar to that found in the literature using RDG approximation for sizing estimation [9, 27, 30, 46]. Nevertheless, the present uncertainties at the wings are much higher, not better than $40 \%$, due to the relatively low spatial resolution to resolve the large radial intensity gradients of light scattering in these regions. Therefore, a slight spatial mismatch among intensity fields could locally alter the effective radius of gyration measurement, generating fluctuating noise. This issue might be improved in a future experiment by imaging with higher spatial resolution, following Kempema and Long [20].

The intensity ratios used in the 2D-MALS computations exhibit excellent line regression with respect to the square of the scattering wave vector magnitude, showing $R^{2}$ of the least-squares fitting greater than 0.9 in most part of the measured field (Fig. 8d-f). For all instantaneous fields of both flames, $R^{2}$ is always above 0.7 , indicating that the present optical setup is properly corrected using Rayleigh scattering and spatial correspondence is suitable.

Typical instantaneous centreline profiles of effective radius of gyration are displayed in Fig. 9 for the $60 \% \mathrm{C}_{2} \mathrm{H}_{4}$ diffusion flame, including the same instants plotted in Fig. 8 . The size of the soot aggregates increases with height above the burner along the centreline until the flame tip, leading to the biggest aggregates at the flame tip. This is an expected behaviour due to the longer residence time in the flame experienced by the soot aggregates [44]. Upstream this region, the sizes decrease due to oxidation [44]. The variations of effective radius of gyration and axial length among centreline profiles are mainly influenced by the flickering flame behaviour.

Figure 10 presents time-averaged effective radius of gyration fields and their standard deviation fields from the 50 and $60 \% \mathrm{C}_{2} \mathrm{H}_{4}$ diffusion flames. The fields are calculated

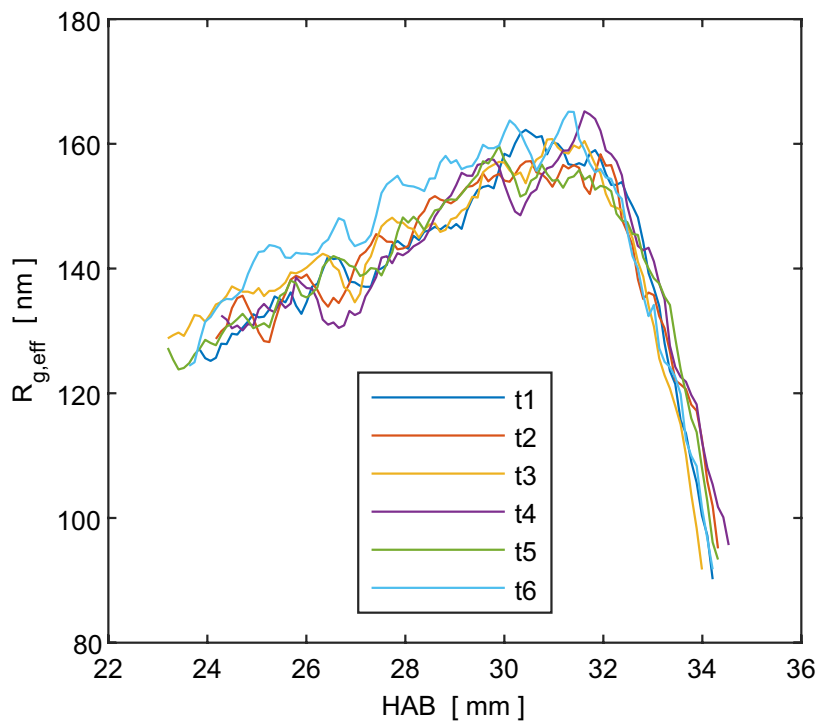

Fig. 9 Examples of single-shot centreline profiles of effective radii of gyration for the diluted $60 \% \mathrm{C}_{2} \mathrm{H}_{4}$ diffusion flame 
from 100 instantaneous effective radius of gyration fields. It is important to mention that the amount of measurements employed in the statistics changes locally (and is accounted for) due to small border oscillations of the flame (flame flickering). Time-averaged values obtained from less than 20 instantaneous measurements are filtered out. The singleshot MALS allows for measurements of two-dimensional fields of fluctuations, which were only possible by scanning point-wise WALS measurements before [33, 34]. In both flames, the biggest soot aggregates are found at the flame tips (Fig. 10a,c). This finding has been also reported by many other researchers for a variety of diffusion flames [e.g., 5, 23, 44]. The averaged soot aggregate sizes in the $60 \% \mathrm{C}_{2} \mathrm{H}_{4}$ diffusion flame are greater than those in the $50 \%$ $\mathrm{C}_{2} \mathrm{H}_{4}$ diffusion flame. This is an expected behaviour due to the longer residence time for the soot to grow by aggregation and agglomeration in the less diluted flame, as reported in the literature [20, 27, 44]. The averaged field for the $60 \%$ $\mathrm{C}_{2} \mathrm{H}_{4}$ diffusion flame is not perfectly axisymmetric due to the insufficient amount of samples to converge the statistics. This flame exhibits about 3 -fold more flickering than the $50 \% \mathrm{C}_{2} \mathrm{H}_{4}$ diffusion flame. For both flames, the greatest fluctuations of the effective radios of gyration are observed at the flame tip and wings. This is caused, not only due to flame flickering, but also due the highest signal gradients leading to bigger errors in the spatial correspondence between signals from different views in these regions. The low spatial resolution in the present measurements, which is a trade-off for a high dynamic spatial range, is not enough to sufficiently resolve aggregation variations in the flame wings of the diffusion flame. We should emphasise that the present single-shot 2D-MALS technique, differently from the existing averaged 2D-MALS, is able to deliver higher-order moments of the effective radios of gyration (e.g., skewness and kurtosis) in weakly unsteady flames. Nevertheless, here the analysis is restricted to the first- and second-order moments (i.e. average and standard deviation), since the amount of the present data would be insufficient to converge statistics of higher order.

The range of the present values of effective radius of gyration are compared with measurements using averaged 2D-MALS technique in $\mathrm{N}_{2}$-diluted laminar $\mathrm{C}_{2} \mathrm{H}_{4}$ coflow laminar diffusion flames from other researching groups. Table 1 reproduces the range of values extracted from the masked 2D fields of effective radius of gyration measured by Ma and Long [27] and by Kempema and Long [20]. Measurements in $40 \% \mathrm{C}_{2} \mathrm{H}_{4}$ diffusion flame from the latter authors are included as a reference of the upper limit values expected according to the flame dilution. From the table, it is possible to observe that the present measurements are within the expected range of averaged effective radius of gyration of soot aggregates for the studied flames. It is worth

Table 1 Averaged effective radius of gyration measured using 2D-MALS techniques in $\mathrm{N}_{2}$-diluted laminar $\mathrm{C}_{2} \mathrm{H}_{4}$ coflow laminar diffusion flames from the literature and from the present work

\begin{tabular}{llll}
\hline & $40 \% \mathrm{C}_{2} \mathrm{H}_{4}$ & $50 \% \mathrm{C}_{2} \mathrm{H}_{4}$ & $60 \% \mathrm{C}_{2} \mathrm{H}_{4}$ \\
\hline Ma and Long [27] & - & - & $50-200$ \\
Kempema and Long [20] & $20-70$ & - & $20-180$ \\
Present work & - & $80-140$ & $80-180$ \\
\hline
\end{tabular}
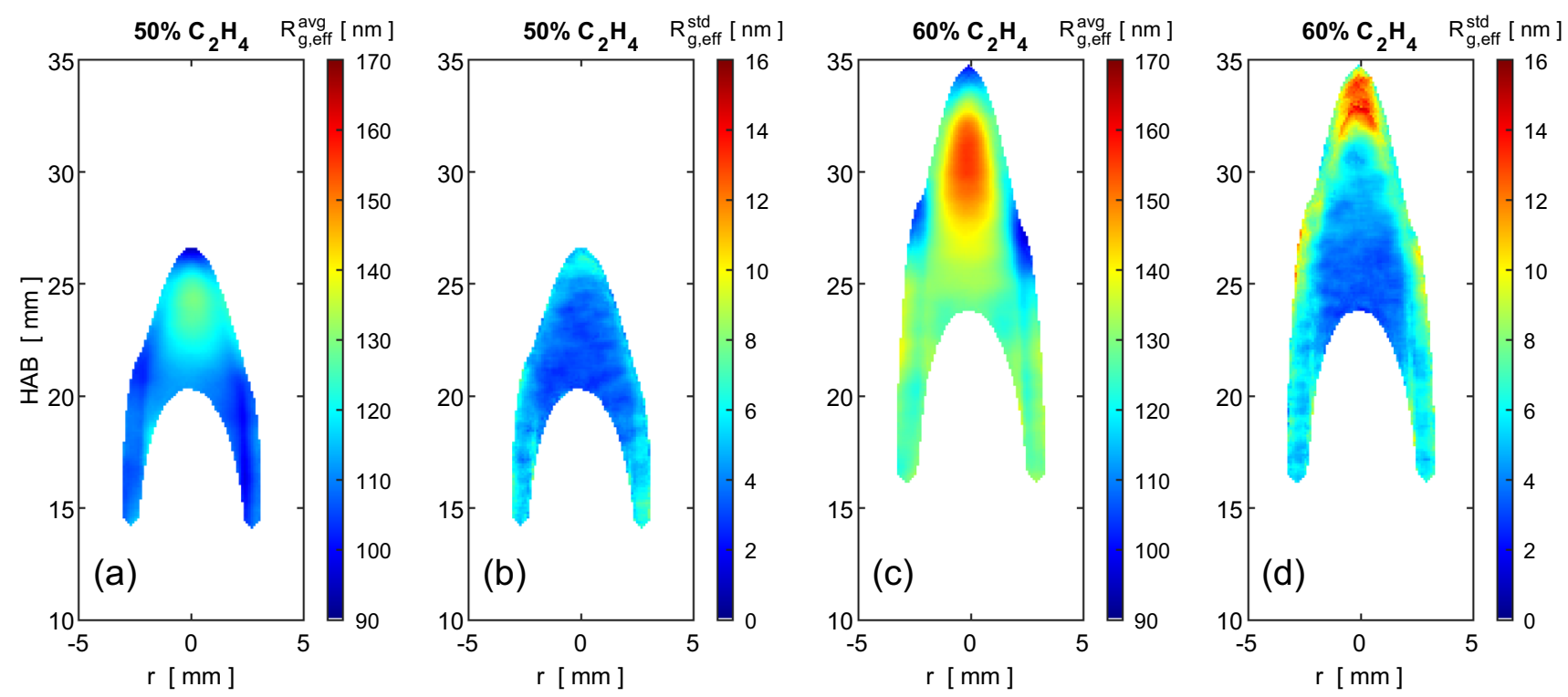

Fig. 10 Two-dimensional fields of (a, c) time-average and (b, d) standard deviation of effective radius of gyration for 50 and $60 \% \mathrm{C}_{2} \mathrm{H}_{4}$ diffusion flames 
mentioning though that effective radii of gyration below 80 are not observed in the present results due to the low acquired scattering signal, as explained previously, and that the radii of gyration reproduced from the literature might be underestimated. Since the latter measurements were evaluated from intensity ratios with respect to ten-degree viewing direction $\left(I_{\text {sca }}\left(10^{\circ}\right) / I_{\text {sca }}(\theta)\right)$, instead of zero-degree direction (cf. eq. 4), the derived effective radii of gyration tend to be biased towards lower values [27, 33].

Effective averaged soot radii of gyration along the centreline of 50 and $60 \%$ flames as a function of the height above the burner are given in Fig. 11. The averaged values are computed based on the time average of single-shot effective radii of gyration (present 2D-MALS technique) and based on the time-averaged light scattered signals, following the work of Altenhoff et al. [1]. The latter technique serves as a target result, because it has been already proved to be an efficient tool for measuring averaged effective radius of gyration by different groups [e.g., 1, 27]. Standard deviation bars are added every fifth measurement location in order to not overcrowd the graph. Excellent agreement is obtained between both approaches, attesting the performance of the singleshot 2D-MALS technique. For both flames, the averaged curves of effective radius of gyration increase smoothly and monotonically towards a peak around 24 and $31 \mathrm{~mm}$ HAB for 50 and $60 \%$ flames, respectively, and then decrease. The decreasing behaviour is a consequence of the soot oxidation at the flame closed tip [5, 23, 44]. The decreasing slopes are less stiff than those found in the instantaneous effective radius of gyration profiles (Fig. 9) as a result of smoothing by the average procedure, where the flame shape change

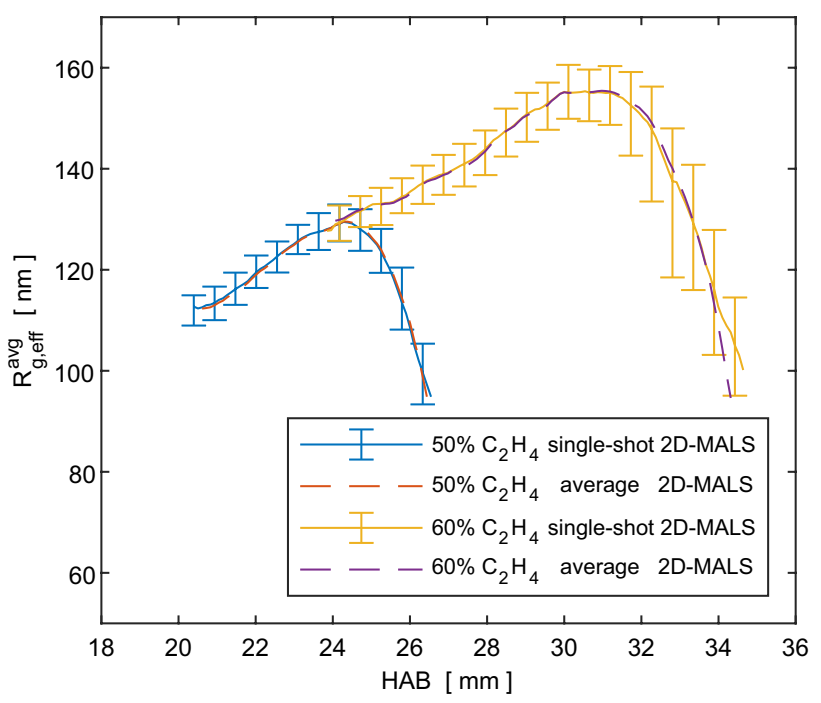

Fig. 11 Comparison of centreline averaged profiles of effective radius of gyration for 50 and $60 \% \mathrm{C}_{2} \mathrm{H}_{4}$ diffusion flames computed from time-averaged single-shot effective radii of gyration and from timeaveraged light scattered signals. Bars refer to one standard deviation (due to flickering) may lead to the contribution of different regions of the flame in the evaluation. The scattered signal diminishes significantly for upstream and downstream positions of the profile, as discussed previously, causing the termination of the measured profiles at both sides. The present results are in very well agreement with the 2D-MALS measurements performed by Ma and Long [27] and Kempema and Long [20] away from the extreme of the flame tip. Their averaged effective radii of gyration of the soot aggregate along the centreline did not decrease upstream, because their diluted $\mathrm{C}_{2} \mathrm{H}_{4}$ diffusion flames were sooting flames, with different flow rates (and flow dynamics) than those from the non-sooting flames of our work.

The present technique allows assess the probability density function (PDF) of effective radius of gyration. Examples of such PDF distributions at $20.5 \mathrm{~mm}$ HAB for the 50\% flame and at 23.8 and $30.5 \mathrm{~mm} \mathrm{HAB}$ for the $60 \%$ flame along the centreline are presented in Fig. 12. The distributions are obtained over a $5 \times 5$ kernel centred at the aforementioned positions for better convergence. Lognormal curves (continuous curves) and time-averaged values (dashed vertical lines) are also plotted for comparison. The distributions of effective radius of gyration follow lognormal curves for both flames (Fig. 12). Lognormal distribution shapes are also observed at the wings of both flames (not presented for sake of space). The curves are in agreement with transmission electric microscope (TEM) measurements reported in the literature for a variety of diluted $\mathrm{C}_{2} \mathrm{H}_{4}$ diffusion flames [20].

\section{Conclusion}

The present work extends the two-dimensional multi-angle light scattering technique for measurements of effective radii of gyration of soot aggregates on a single-shot basis. A single-pulse laser is combined with six CCD cameras circularly positioned from 10 to $90^{\circ}$ for synchronous signal recordings. The approach is demonstrated in $\mathrm{N}_{2}$-diluted 50 and $60 \% \mathrm{C}_{2} \mathrm{H}_{4}$ coflow laminar diffusion flames with small flickering. Dissimilarities of the optical setups at the six different viewing directions are successfully corrected based on the light scattering from pure gases. Spatial distortions in the camera images are sufficiently corrected based on the pinhole model followed by a minor 2 nd-order polynomial correction to take into account the misalignment between the calibration target and the light sheet and the non-homogeneous refractive index along the light path.

Results show single-shot fields of effective radii of gyration of soot aggregates with high $R^{2}$ values of least-squares curve fittings. Instantaneous centreline profiles of effective radii of gyration present monotonic increase of sizes with height above the burner upstream the flame tip due to aggregation and agglomeration of soot clusters. At the 


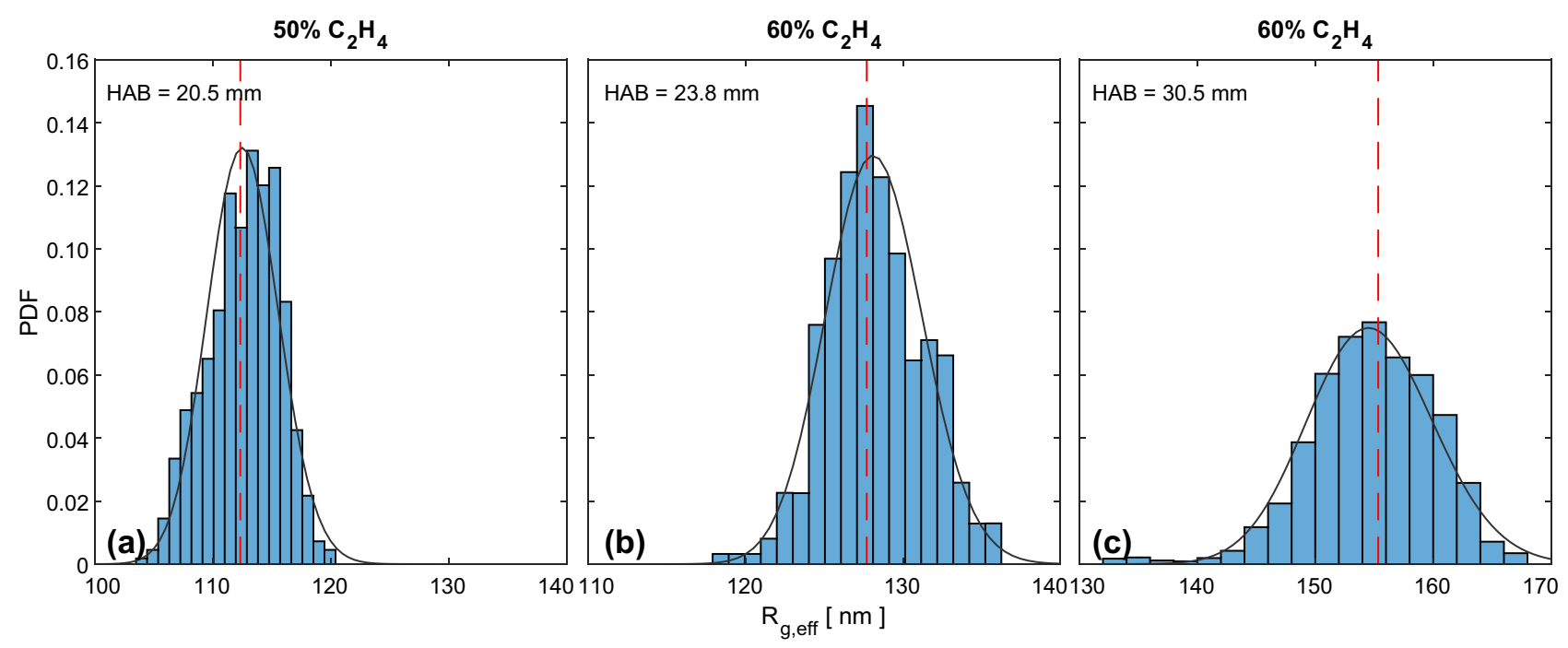

Fig. 12 Probability density function of effective radius of gyration along the centreline at different heights above the burner for 50 and $60 \% \mathrm{C}_{2} \mathrm{H}_{4}$ diffusion flames. The average value is displayed as a vertical dashed line and probabilistic distribution fits as continuous curves

flame tip, effective radii of gyration decrease with HAB due to oxidation for both flames under study. Time-averaged fields of effective radii of gyration evaluated by sing-shot 2D-MALS remarkably agree with those computed by averaged 2D-MALS [27], technique already established in the literature. The range of aggregate sizes and the behaviour of the centreline profiles of the current work are in line with other researchers. The proposed technique can additionally provide fields of higher-order statistics, such as standard deviation, skewness and kurtosis as well as probability density function maps of effective radii of gyration of aggregates. The present standard deviation fields show the greatest values at the flame wings and tip, whereas PDF distributions are characterised by lognormal curves for both studied flames. In summary, the present single-shot 2D-MALS prove to be a tool for acquiring instantaneous full-regionof-interest snapshots of the effective radios of gyration of aggregates in weakly unsteady diffusion flames and might be extended to more complex combustion processes.

Acknowledgements The German Research Foundation (DFG) funding within the priority program 'Nanoparticle Synthesis in Spray Flames, SpraySyn: Measurement, Simulation, Processes' (SPP1980, BE 3773/3 and KR 3684/12) is gratefully acknowledged. The authors thank the colleagues Benoît Fond for the fruitful discussions, Moritz Stelter for assisting with the experiments, and Katharina Zähringer for lending some of the optical components used.

Funding Open Access funding enabled and organized by Projekt DEAL.

Open Access This article is licensed under a Creative Commons Attribution 4.0 International License, which permits use, sharing, adaptation, distribution and reproduction in any medium or format, as long as you give appropriate credit to the original author(s) and the source, provide a link to the Creative Commons licence, and indicate if changes were made. The images or other third party material in this article are included in the article's Creative Commons licence, unless indicated otherwise in a credit line to the material. If material is not included in the article's Creative Commons licence and your intended use is not permitted by statutory regulation or exceeds the permitted use, you will need to obtain permission directly from the copyright holder. To view a copy of this licence, visit http://creativecommons.org/licenses/by/4.0/.

\section{References}

1. M. Altenhoff, S. Aßmann, J.F.A. Perlitz, F.J.T. Huber, S. Will, Soot aggregate sizing in an extended premixed flame by high-resolution two-dimensional multi-angle light scattering (2D-MALS). Appl. Phys. B 125, 176 (2019)

2. W.H. Beck, E. Stockman, S.H. Zaidi, R.B. Miles, Rayleigh scattering measurements for obtaining spatially-resolved absolute gas densities in large scale facilities, in: 44th AIAA Aerospace Sciences Meeting and Exhibit, Reno, Nevada. pp. AIAA 2006-835 (2006)

3. H. Bockhorn, Soot Formation in Combustion: Mechanisms and Models, vol. 59 (Springer Science and Business Media, Berlin, 2013).

4. T.C. Bond, S.J. Doherty, D.W. Fahey, P.M. Forster, T. Berntsen, B.J. DeAngelo, M.G. Flanner, S. Ghan, B. Kärcher, D. Koch et al., Bounding the role of black carbon in the climate system: a scientific assessment. J. Geophys. Res. 118, 5380-5552 (2013)

5. M.L. Botero, Y. Sheng, J. Akroyd, J. Martin, J.A.H. Dreyer, W. Yang, M. Kraft, Internal structure of soot particles in a diffusion flame. Carbon 141, 635-642 (2019)

6. M.Y. Choi, A. Hamins, G.W. Mulholland, T. Kashiwagi, Simultaneous optical measurement of soot volume fraction and temperature in premixed flames. Combust. Flame 99, 174-186 (1994)

7. M.Y. Choi, G.W. Mulholland, A. Hamins, T. Kashiwagi, Comparisons of the soot volume fraction using gravimetric and light extinction techniques. Combust. Flame 1, 161-169 (1995) 
8. W.A. England, An in situ X-ray small angle scattering study of soot morphology in flames. Combust. Sci. Technol. 46, 83-93 (1986)

9. T.L. Farias, M.G. Carvalho, Ü.Ö. Köylü, G.M. Faeth, Computational evaluation of approximate Rayleigh-Debye-Gans/FractalAggregate theory for the absorption and scattering properties of soot. J. Heat Transf. 117, 152-159 (1995)

10. S. Gangopadhyay, I. Elminyawi, C.M. Sorensen, Optical structure factor measurements of soot particles in a premixed flame. Appl. Opt. 30, 4859-4864 (1991)

11. P.S. Greenberg, J.C. Ku, Soot volume fraction imaging. Appl. Opt. 36, 5514-5522 (1997)

12. R. Hartley, A. Zisserman, Multiple View Geometry in Computer Vision (Cambridge University Press, Cambridge, 2003).

13. B.S. Haynes, H. Jander, H.G. Wagner, Optical studies of sootformation processes in premixed flames. Berichte der Bunsengesellschaft für Physikalische Chemie 84, 585-592 (1980)

14. M.V. Heitor, A.L.N. Moreira, Thermocouples and sample probes for combustion studies. Progress Energy Combust. Sci. 19, 259278 (1993)

15. J.P. Hessler, S. Seifert, R.E. Winans, T.H. Fletcher, Small-angle $\mathrm{X}$-ray studies of soot inception and growth. Faraday Discuss. 119, 395-407 (2002)

16. E.J. Highwood, R.P. Kinnersley, When smoke gets in our eyes: the multiple impacts of atmospheric black carbon on climate, air quality and health. Environ. Int. 32, 560-566 (2006)

17. F.J.T. Huber, S. Will, Characterization of a silica-aerosol in a sintering process by wide-angle light scattering and principal component analysis. J. Aerosol Sci. 119, 62-76 (2018)

18. A.R. Jones, Light scattering for particle characterization. Progress Energy Combust. Sci. 25, 1-53 (1999)

19. A.W. Kandas, I.G. Senel, Y. Levendis, A.F. Sarofim, Soot surface area evolution during air oxidation as evaluated by small angle X-ray scattering and $\mathrm{CO}_{2}$ adsorption. Carbon 43, 241-251 (2005)

20. N. Kempema, M.B. Long, Combined optical and TEM investigations for a detailed characterization of soot aggregate properties in a laminar coflow diffusion flame. Combust. Flame 64, 373-385 (2016)

21. Ü.Ö. Köylü, Y. Xing, D.E. Rosner, Fractal morphology analysis of combustion-generated aggregates using angular light scattering and electron microscope images. Langmuir 11, 4848-4854 (1995)

22. C.B. Lee, W. Lee, K.C. Oh, H.D. Shin, J.K. Yoon, Sooting and non-sooting propylene diffusion flames with irradiation of laser light. Combust. Explos. Shock Waves 42, 688-695 (2006)

23. Z. Li, L. Qiu, X. Cheng, Y. Li, H. Wu, The evolution of soot morphology and nanostructure in laminar diffusion flame of surrogate fuels for diesel. Fuel 211, 517-528 (2018)

24. O. Link, D.R. Snelling, K.A. Thomson, G.J. Smallwood, Development of absolute intensity multi-angle light scattering for the determination of polydisperse soot aggregate properties. Proc. Combust. Instit. 33, 847-854 (2011)

25. M. Lippmann, Toxicological and epidemiological studies of cardiovascular effects of ambient air fine particulate matter $\left(\mathrm{PM}_{2.5}\right)$ and its chemical components: coherence and public health implications. Crit. Rev. Toxicol. 44, 299-347 (2014)

26. F. Liu, K.A. Thomson, G.J. Smallwood, Soot temperature and volume fraction retrieval from spectrally resolved flame emission measurement in laminar axisymmetric coflow diffusion flames: effect of self-absorption. Combust. Flame 160, 16931705 (2013)

27. B. Ma, M.B. Long, Combined soot optical characterization using 2-D multi-angle light scattering and spectrally resolved line-of-sight attenuation and its implication on soot color-ratio pyrometry. Appl. Phys. B 117, 287-303 (2014)
28. H.A. Michelsen, Probing soot formation, chemical and physical evolution, and oxidation: A review of in situ diagnostic techniques and needs. Proc. Combust. Instit. 36, 717-735 (2017)

29. H.A. Michelsen, C. Schulz, G.J. Smallwood, S. Will, Laserinduced incandescence: particulate diagnostics for combustion, atmospheric, and industrial applications. Progress Energy Combust. Sci. 51, 2-48 (2015)

30. F. Migliorini, K.A. Thomson, G.J. Smallwood, Investigation of optical properties of aging soot. Appl. Phys. B 104, 273-283 (2011)

31. J.B.A. Mitchell, J.L. Le Garrec, A.I. Florescu-Mitchell, S. Di Stasio, Small-angle neutron scattering study of soot particles in an ethylene-air diffusion flame. Combust. Flame 145, 80-87 (2006)

32. M. Ni, H. Zhang, F. Wang, Z. Xie, Q. Huang, J. Yan, K. Cen, Study on the detection of three-dimensional soot temperature and volume fraction fields of a laminar flame by multispectral imaging system. Appl. Therm. Eng. 96, 421-431 (2016)

33. H. Oltmann, J. Reimann, S. Will, Wide-angle light scattering (WALS) for soot aggregate characterization. Combust. Flame 157, 516-522 (2010)

34. H. Oltmann, J. Reimann, S. Will, Single-shot measurement of soot aggregate sizes by wide-angle light scattering (WALS). Appl. Phys. B 106, 171-183 (2012)

35. F.X. Ouf, J. Yon, P. Ausset, A. Coppalle, M. Maillé, Influence of sampling and storage protocol on fractal morphology of soot studied by transmission electron microscopy. Aerosol Sci. Technol. 44, 1005-1017 (2010)

36. R. Puri, T.F. Richardson, R.J. Santoro, R.A. Dobbins, Aerosol dynamic processes of soot aggregates in a laminar ethene diffusion flame. Combust. Flame 92, 320-333 (1993)

37. B. Quay, T.W. Lee, T. Ni, R.J. Santoro, Spatially resolved measurements of soot volume fraction using laser-induced incandescence. Combust. Flame 97, 384-392 (1994)

38. R.J. Santoro, H.G. Semerjian, R.A. Dobbins, Soot particle measurements in diffusion flames. Combust. Flame 51, 203-218 (1983)

39. F. Scarano, L. David, M. Bsibsi, D. Calluaud, S-PIV comparative assessment: image dewarping + misalignment correction and pinhole+ geometric back projection. Exp. Fluids 39, 257$266(2005)$

40. R. Schlüßler, J. Czarske, A. Fischer, Uncertainty of flow velocity measurements due to refractive index fluctuations. Opt. Lasers Eng. 54, 93-104 (2014)

41. C. Schulz, T. Dreier, M. Fikri, H. Wiggers, Gas-phase synthesis of functional nanomaterials: challenges to kinetics, diagnostics, and process development. Proc. Combust. Instit. 37, 83-108 (2018)

42. C. Schulz, B.F. Kock, M. Hofmann, H. Michelsen, S. Will, B. Bougie, R. Suntz, G. Smallwood, Laser-induced incandescence: recent trends and current questions. Appl. Phys. B 83, 333 (2006)

43. C.R. Shaddix, J.E. Harrington, K.C. Smyth, Quantitative measurements of enhanced soot production in a flickering methane/ air diffusion flame. Combust. Flame 99, 723-732 (1994)

44. M.D. Smooke, M.B. Long, B.C. Connelly, M.B. Colket, R.J. Hall, Soot formation in laminar diffusion flames. Combust. Flame 143, 613-628 (2005)

45. D.R. Snelling, K.A. Thomson, G.J. Smallwood, Ö.L. Gülder, Two-dimensional imaging of soot volume fraction in laminar diffusion flames. Appl. Opt. 38, 2478-2485 (1999)

46. C.M. Sorensen, Light scattering by fractal aggregates: a review. Aerosol Sci. Technol. 35, 648-687 (2001)

47. A. Stella, G. Guj, J. Kompenhans, M. Raffel, H. Richard, Application of particle image velocimetry to combusting flows: 
design considerations and uncertainty assessment. Exp. Fluids 30, 167-180 (2001)

48. T.F. Stocker, D. Qin, G.K. Plattner, M. Tignor, S.K. Allen, J. Boschung, A. Nauels, Y. Xia, V. Bex, P.M. Midgley, et al., Climate change 2013: The physical science basis. Contribution of working group I to the fifth assessment report of the intergovernmental panel on climate change, 1535 (2013)

49. R. Strobel, S.E. Pratsinis, Flame aerosol synthesis of smart nanostructured materials. J. Mater. Chem. 17, 4743-4756 (2007)

50. Z. Tan, Air Pollution and Greenhouse Gases: From Basic Concepts to Engineering Applications for Air Emission Control (Springer, Berlin, 2014).

51. R.L. Vander Wal, M.Y. Choi, Pulsed laser heating of soot: morphological changes. Carbon 37, 231-239 (1999)

52. C. Vanselow, A. Fischer, Influence of inhomogeneous refractive index fields on particle image velocimetry. Opt. Lasers Eng. 107, 221-230 (2018)

53. H. Wang, B. Zhao, B.E. Wyslouzil, K.A. Streletzky, Small-angle neutron scattering of soot formed in laminar premixed ethylene flames. Proc. Combust. Instit. 29, 2749 (2002)

54. B. Wieneke, Stereo-PIV using self-calibration on particle images. Exp. Fluids 39, 267-280 (2015)
55. S. Will, S. Schraml, A. Leipertz, Two-dimensional soot-particle sizing by time-resolved laser-induced incandescence. Opt. Lett. 20, 2342-2344 (1995)

56. C. Willert, Stereoscopic digital particle image velocimetry for application in wind tunnel flows. Meas. Sci. Technol. 8, 1465 (1997)

57. G.D. Yoder, P.K. Diwakar, D.W. Hahn, Assessment of soot particle vaporization effects during laser-induced incandescence with time-resolved light scattering. Appl. Opt. 44, 4211-4219 (2005)

58. B. Zhao, K. Uchikawa, H. Wang, A comparative study of nanoparticles in premixed flames by scanning mobility particle sizer, small angle neutron scattering, and transmission electron microscopy. Proc. Combust. Instit. 31, 851-860 (2007)

Publisher's Note Springer Nature remains neutral with regard to jurisdictional claims in published maps and institutional affiliations. 